\title{
DNA-based discrimination and frequency of phytoplasma infection in the two hawthorn-feeding species, Cacopsylla melanoneura and Cacopsylla affinis, in northwestern Italy
}

\author{
R. Tedeschi ${ }^{1 *}$ and F. Nardi ${ }^{2}$ \\ ${ }^{1}$ DIVAPRA - Entomologia e Zoologia applicate all'Ambiente 'C. Vidano', \\ Facoltà di Agraria, Università degli Studi di Torino. Via Leonardo da \\ Vinci 44, 10095 Grugliasco (TO), Italy: ${ }^{2}$ Dipartimento di Biologia Evolutiva, \\ Università di Siena. Via Aldo Moro 2, 53100 Siena, Italy
}

\begin{abstract}
A molecular tool, focused on the mitochondrial Control Region (CR), was developed to discriminate the two hawthorn-feeding psyllid species, Cacopsylla melanoneura (Förster) and C. affinis (Löw), and to estimate their frequencies in mixed populations. The test was carried out in paired and single-tube amplifications and validated analysing 52 male specimens previously determined by morphological analysis. The frequencies of the two species in mixed populations in the Aosta Valley (northwestern Italy) were analysed. The presence and type of 16SrX-group phytoplasmas was detected by nested PCR and RFLP tests in both species. C. melanoneura was the predominant species (86.5\%; 80.4-91.2 CI); of these, $0.9 \%$ of the samples were positive for ' $\mathrm{Ca}$. Phytoplasma mali' and $1.8 \%$ for 'Ca. Phytoplasma pyri'. One of 21 specimens of C. affinis was positive for 'Ca. Phytoplasma pyri'. The test also allowed us to identify two genetic variants of C. melanoneura, depending on the presence or absence of a $56 \mathrm{bp}$ indel; these were named WI (with indel) and WOI (without indel), respectively. Further analyses were carried out on C. melanoneura specimens collected in apple orchards at six different locations in northern Italy where different levels of transmission efficiency have been described. Our preliminary observations suggest that some differences might exist between the two genetic variants in their ability to transmit phytoplasmas and to colonise different host plants.
\end{abstract}

Keywords: apple, Cacopsylla affinis, Cacopsylla melanoneura, hawthorn, molecular tools, phytoplasmas

(Accepted 1 February 2010)

\section{Introduction}

Cacopsylla melanoneura (Förster) and Cacopsylla affinis (Löw) are two psyllid species that live primarily on Crataegus

*Author for correspondence

Fax: + 390112368675

E-mail: rosemarie.tedeschi@unito.it spp., although C. melanoneura has also been implicated in the transmission of phytoplasmas to apple trees.

C. melanoneura is a Holo-Palaearctic species found throughout Europe, with the exception of the northernmost provinces of Norway and eastern Fennoscandia. It has always been reported as a primarily hawthorn-feeding species (Lal, 1934; Ossiannilsson, 1992); nevertheless, it has been shown to use both apple and hawthorn plants as 
reproductive hosts, on which it completes one generation per year. After the emergence of new adults, it migrates to conifers for estivation and overwintering (Lauterer, 1999; Tedeschi et al., 2002, 2009; Čermák \& Lauterer, 2008; Mayer \& Gross, 2007).

C. melanoneura received significant attention in recent years as the main vector, in northwestern Italy, of the phytoplasma 'Candidatus Phytoplasma mali' (Tedeschi et al., 2003; Tedeschi \& Alma, 2004), the causal agent of the apple proliferation (AP) disease that induces serious economic losses in apple production via reduction in size and tasteless fruits. Other suitable vectors of the phytoplasma are the congeneric C. picta (Förster) (Syn. C. costalis) (Frisinghelli et al., 2000; Jarausch et al., 2003) and the leafhopper Fieberiella florii (Stål) (Tedeschi \& Alma, 2006).

C. affinis is an Euro-Caucasian species and is present in most European countries, where it can be observed on free-growing shrubs and in woodland ecotones and scrub. It is univoltine and oligophagous on Crataegus spp., predominantly feeding on Cr. monogyna. C. affinis has never been considered as a noxious species for hawthorn plants, possibly as a consequence of the low population densities that are normally recorded. The species was shown to host phytoplasmas of the AP group (Tedeschi et al., 2009), but its role as a vector still needs to be assessed.

The two species share a similar life cycle and morphology. The presence period on hawthorn plants is similar, and likewise adults hibernate on various plant species, above all conifers (Lauterer, 1999). Furthermore, while C. melanoneura populations on apple trees are apparently pure (Lauterer, 1999; R. Tedeschi, personal observations), the two species are often observed to form mixed populations on hawthorn shrubs (Lauterer, 1999; Tedeschi et al., 2009). Males of the two species can be discriminated by morphological observation of the apical part of the aedeagus and the shape of the parameres, although this is not readily accomplished by the non-specialist, and the latter character shows some inter-individual variability. Females, on the other hand, cannot be reliably distinguished based on morphology.

The recent finding of ' $\mathrm{C} a$. Phytoplasma mali' and other fruit tree phytoplasmas in both $C$. melanoneura and C. affinis males collected on Cr. monogyna, a known source of inoculum (Tedeschi et al., 2009), revealed the necessity to investigate the biology of the two species, their host preferences and their relative role as vectors in more detail.

The primary aim of the present work is to develop and validate a molecular tool to readily discriminate between C. melanoneura and C. affinis. Furthermore, the test is applied in a preliminary screening of field samples (i) to estimate the frequency of the two species in mixed populations on hawthorn and (ii) to estimate the frequency of two genetic variants of $C$. melanoneura collected on hawthorn and apple trees and their relation with $16 \mathrm{SrX}$-group phytoplasmas.

In the development of the molecular test, we focused on the control region (CR) of the mitochondrial genome, as it is highly variable but at the same time bounded by conserved regions (srRNA and $\mathrm{tRNA}-\mathrm{I} / \mathrm{Q} / \mathrm{M}$ ) that are suitable for cross-species primer design. Furthermore, the peculiar structure of the region and its structural flexibility maximise the possibility of observing major indels, which are easily targeted using PCR-based tests.

\section{Material and methods}

\section{Insect samples and DNA extraction}

C. melanoneura-C. affinis mixed samples were collected during 2006 and 2008 on wild Crataegus monogyna Jacquin in the Aosta Valley (northwestern Italy) using beat trays. A tree limb was struck several times to dislodge psyllids onto white plastic trays $(250 \times 350 \mathrm{~mm})$ held beneath. Insects were carried to the laboratory and preserved at $-80^{\circ} \mathrm{C}$ for molecular analysis. A subsample composed only of males was preserved in ethanol and determined to the species level by morphological analysis.

Additional specimens of C. melanoneura were collected, and preserved as above, on apple plants from different regions of northern Italy.

DNA was purified from individual psyllids using either the Wizard ${ }^{\circledR}$ SV Genomic DNA Purification System (Promega) or a protocol adapted from Marzachì et al. (1998) and previously applied in psyllids (Tedeschi et al., 2002). The commercial kit was used for the initial sequencing experiments; the latter procedure, routinely used for phytoplasma detection, was applied in the screening.

\section{Sequencing of Cacopsylla melanoneura and Cacopsylla affinis control region}

The complete mitochondrial genome of representative Hemipterans (seven in six available families; NC_005944, NC_002609, NC_006899, NC_006158, NC_006280, NC_ 005939, NC_006157) was obtained from GenBank, and the two regions that surround the CR (srRNA and tRNA-I/Q/ $\mathrm{M})$ were aligned. Two primers were designed to amplify the entire CR and terminal stretches of srRNA and tRNA-I/Q/ M: Ps_srRNA_J: ACCGCAACGGCTGGCAC, Ps_ILE_N: ATCAAAATAATCCTTTTTCAGGCA, corresponding to positions 12556/12572 and 13349/13371 in the complete mitochondrial genome of Pachypsylla venusta (NC_006157).

Briefly, suitable conserved regions were identified on the aligned sequences, and multiple primers were manually designed. The best pair was selected by calculating binding properties as implemented in the software Primer3 (ver. 0.4.0, available at http://frodo.wi.mit.edu/primer3/).

PCR reactions were performed in $20 \mu \mathrm{l}$ reaction volume containing $1.5 \mu \mathrm{l}$ of purified DNA template, $0.5 \mu \mathrm{M}$ each primer, $200 \mu \mathrm{M}$ dNTP mix, $1 \times$ PCR buffer, $2 \mathrm{mM} \mathrm{MgCl}_{2}$ and $0.5 \mathrm{U}$ of Platinum ${ }^{\circledR}$ Taq DNA Polymerase (Invitrogen Ltd, Paisley, UK). A touchdown cycling profile was used: initial denaturation of $94^{\circ} \mathrm{C}$ for 3 min followed by 37 cycles of $94^{\circ} \mathrm{C}$ for $1 \mathrm{~min}, 65^{\circ} \mathrm{C}$ (decreasing $1^{\circ} \mathrm{C}$ per cycle in the initial nine cycles) for $70 \mathrm{~s}, 72^{\circ} \mathrm{C}$ for $90 \mathrm{~s}$.

Amplified products in the range of $800-900 \mathrm{bp}$ were gel purified using the Wizard ${ }^{\circledR}$ SV Gel and PCR clean up system kit (Promega) and sequenced, with external primers, at the Genelab commercial facility (Santa Maria di Galeria, Rome) using proprietary chemistry on a ABI PRISM 3100 Genetic Analyzer (Applied Biosystems, Foster City, CA, USA). Electropherograms were manually corrected and assembled using Sequencher (Gene Codes). Due to the inability of sequencing reactions to cross an intervening long poly-T run, the final assembly was conducted by assembling the final poly-T run obtained with primer Ps_srRNA_J with the final poly-A run obtained with primer Ps_ILE_N. The presence of a possible intervening sequence between the two homopolymer runs was excluded by amplifying across 


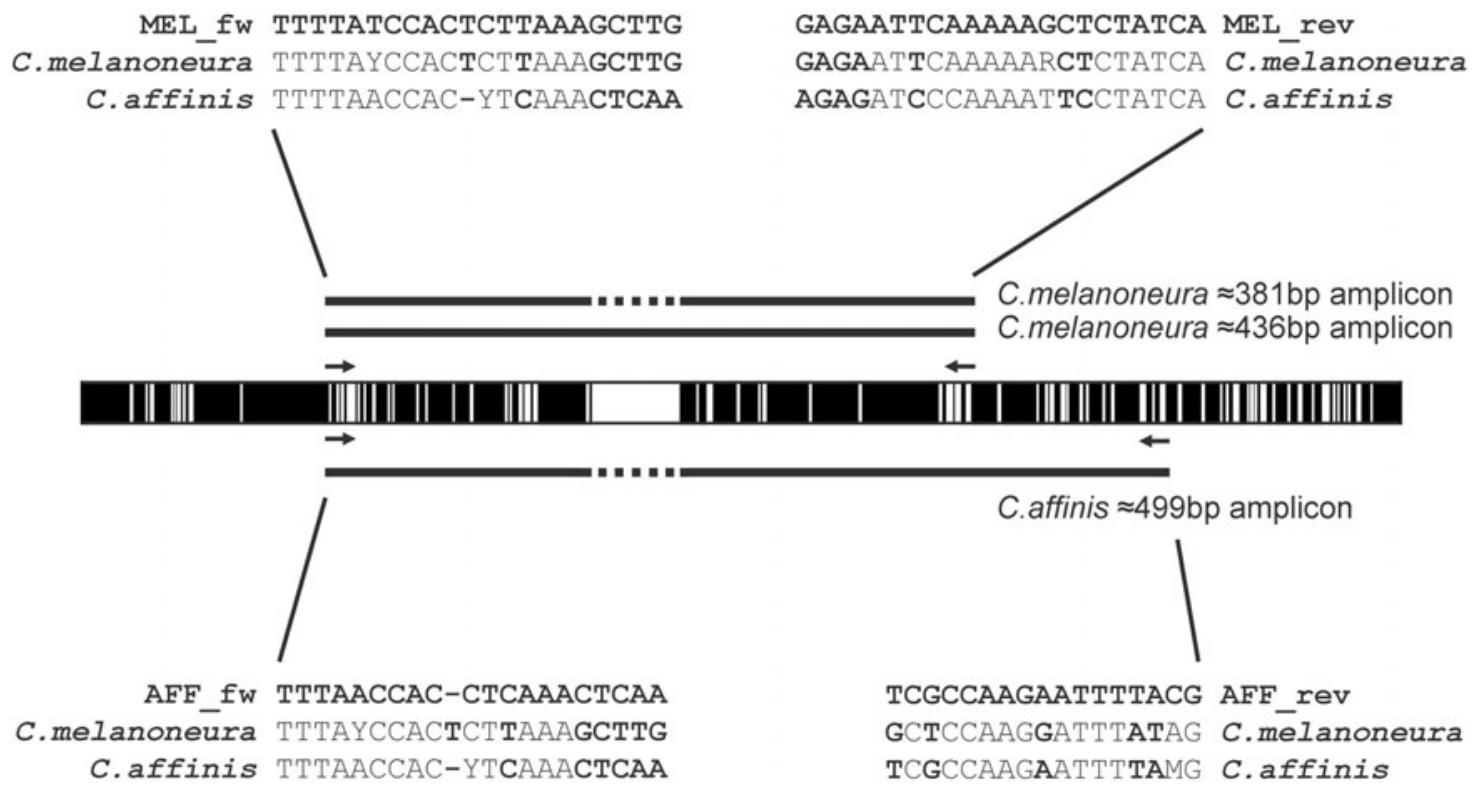

Fig. 1. PCR based test for species discrimination. The central bar represents the sequenced fragment, with variable positions indicated in white. The position of species specific primers and amplicons is shown. Nucleotides that differ between the two species at primer binding sites are in bold.

with neighbouring primers (data not shown) and confirming that the length of the amplicon was as expected assuming no intervening sequence. The number of thymines in the central poly-T run, whose length could not be unambiguously assessed ( $\pm 2 \mathrm{bp}$ ), was set to 23 .

Sequences were obtained from each of six morphologically determined males of each of both species and manually aligned.

\section{PCR-based test for species discrimination}

Two primer pairs were designed to specifically amplify segments of the $C R$ in either $C$. melanoneura or $C$. affinis. Primers were manually designed on suitable sequence segments and their binding properties were calculated using Primer3. Species specificity was achieved by positioning the $3^{\prime}$ end of each primer in short stretches that are conserved within each species and different between them (fig. 1). Expected amplicon length for primer pair MEL_fw: TTTTATCCACTCTTAAAGCTTG/MEL_rev: TGATAGAGCTTTTTGAATTCTC is $436 \mathrm{bp}$ or $381 \mathrm{bp}$ in C. melanoneura (depending on the presence/absence of a long indel, see below) while no amplification is expected in C. affinis. Similarly, expected length for pair AFF_fw: TTTAACCACCTCAAACTCAA/AFF_rev: CGTAAAATTCTTGGCGA is $499 \mathrm{bp}$ in $C$. affinis with no amplification expected in C. melanoneura.

Amplifications were performed in $20 \mu \mathrm{l}$ reactions containing $1.5 \mu \mathrm{l}$ of DNA template, $0.5 \mu \mathrm{M}$ each primer, $200 \mu \mathrm{M}$ dNTP mix, $1 \times$ PCR buffer, $2 \mathrm{mM} \mathrm{MgCl}_{2}$ and $0.5 \mathrm{U}$ of Platinum ${ }^{\circledR}$ Taq DNA Polymerase (Invitrogen Ltd, Paisley, UK). Cycling conditions were as follows: an initial denaturation of $94^{\circ} \mathrm{C}$ for $3 \mathrm{~min}$ followed by 35 cycles of $94^{\circ} \mathrm{C}$ for $1 \mathrm{~min}$, $55^{\circ} \mathrm{C}$ for $70 \mathrm{~s}$, and $72^{\circ} \mathrm{C}$ for $90 \mathrm{~s}$. Amplification products were separated via gel electrophoresis $\left(2 \%\right.$ agarose, $10 \mathrm{~V} \mathrm{~cm}^{-1}$, $1 \times \mathrm{TBE}$ as migration buffer) and visualized using ethidium bromide staining under UV light. The approximate size of PCR products was determined against a molecular ladder (Sigma Aldrich, USA). This same test was carried out in paired tubes (one reaction using primers MEL_fw/MEL_rev and a second reaction using primers AFF_fw/AFF_rev) and in single-tube amplification (with the four primers). In the latter case, a touch-down PCR protocol was applied as described above. The test was performed on the same DNA used for initial sequencing (six individuals for each of the two species) and on 40 additional male specimens, morphologically determined to belong to one of the two species, to check for species specificity and consistency in the results.

Once validated, the tests were performed on a total of 170 specimens collected in the years 2006-2008 on hawthorn plants to assess the frequency of each species in a mixed population.

The test was also performed on 121 specimens collected in apple orchards at six different locations in northern Italy, and thus preliminarily assigned to C. melanoneura to assess the frequency of the $56 \mathrm{bp}$ indel.

\section{Phytoplasma detection}

In order to assess the possibility that C. melanoneura and C. affinis could be infected by $16 \mathrm{SrX}$-group phytoplasmas, molecular analyses were applied to verify the presence of these bacteria in most of the individuals tested for species discrimination. The presence and type of phytoplasma were detected, in DNA purified from single individuals, using a PCR-RFLP test. A first round of amplification was performed using phytoplasma universal primer pair P1/P7 (Schneider et al., 1995), and a 1:40 dilution of the product was subject to a second round of amplification with nested primers fO1/rO1 (Lorenz et al., 1995) specific for AP-group phytoplasmas. Reaction conditions were as in the original papers. Amplification products were analysed by gel 


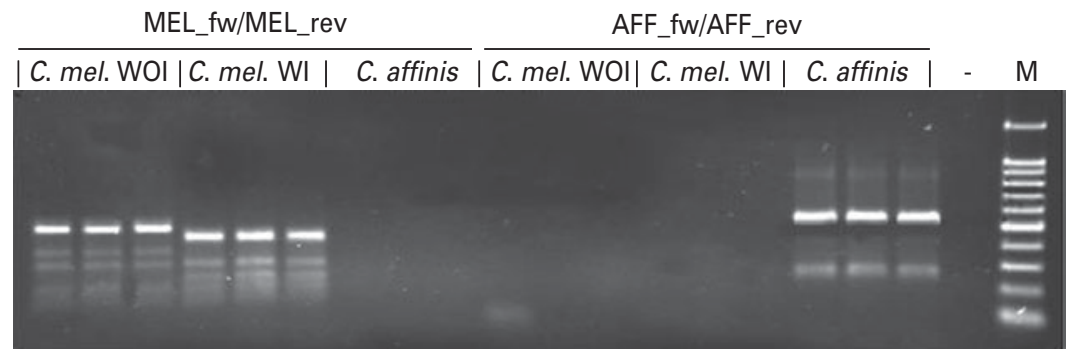

Fig. 2. Products of PCR analysis amplified in paired tubes with the primers MEL_fw/MEL_rev or AFF_fw/AFF_rev from DNA extracted from psyllids previously morphologically determined to belong to the two species Cacopsylla melanoneura and Cacopsylla affinis; -, water; M, Gene Ruler ${ }^{\mathrm{TM}} 100$ bp DNA Ladder (Fermentas).

electrophoresis. Specific AP-group phytoplasma profiles were obtained by RFLP analysis with endonucleases SspI and RsaI, digesting seven microliters of the amplification product with $3 \mathrm{U}$ of $S s p \mathrm{I}$ and $5 \mathrm{U}$ of $\mathrm{RsaI}$ at $37^{\circ} \mathrm{C}$ for three hours.

C. melanoneura individuals collected in apple orchards in six different localities of northern Italy were also tested for phytoplama infection. Moreover, in order to check any correlation between the $56 \mathrm{bp}$ indel observed in some individuals of $C$. melanoneura and the presence of $16 \mathrm{SrX}$ phytoplasmas, another 63 specimens collected in apple orchards and previously proved to be positive for ' $\mathrm{C}$. Phytoplasma mali' were tested with MEL_fw/MEL_rev.

\section{Results}

Sequencing of Cacopsylla melanoneura and Cacopsylla affinis control region

The 12 sequences, corresponding to positions 12583/ 13332 in $P$. venusta, were aligned manually to give a final dataset of $881 \mathrm{bp}$, with 114 variable positions and a large indel of $56 \mathrm{bp}$. The six C. melanoneura individuals are characterized by six different haplotypes that differ at six variable sites $(0.7 \%$ of aligned positions) and one single large insertion of $56 \mathrm{bp}$ that was found in four haplotypes out of six. The six $C$. affinis individuals host three different haplotypes, with six variable sites $(0.7 \%)$ overall. There are 102 fixed point mutations (12\%) between the two species.

The partitioning of genetic differentiation, very limited inside species and fairly large between species, is suitable for the development of DNA-based methods of species discrimination.

\section{Validation of the PCR-based test for species discrimination}

Amplifications performed in paired tubes (MEL_fw/ MEL_rev or AFF_fw/AFF_rev) always gave clear and consistent amplification products of the expected size in the target species and no amplification in the non-target species (fig. 2). Amplifications performed in a single tube with the four primers mostly gave a single product of the expected size (fig. 3). Few dubious profiles were obtained in single tube amplifications of old samples that suffered from many handlings, most likely as a consequence of badly preserved starting material. In all other cases, the signal was clearly interpretable. Amplicon length, as observed from the gel, consistently appeared to be slightly longer than expected.

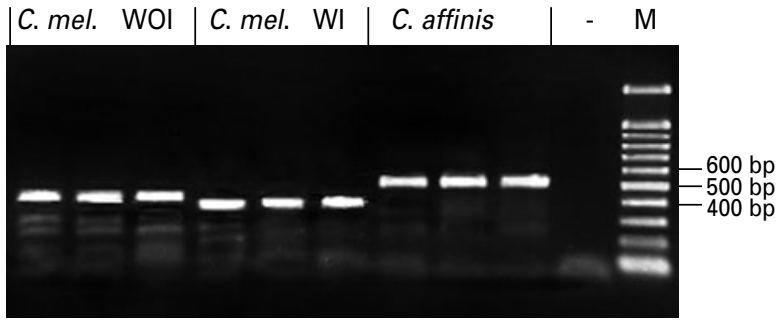

Fig. 3. Products of PCR analysis amplified in single tube with the four primers MEL_fw/MEL_rev and AFF_fw/AFF_rev from DNA extracted from psyllids previously morphologically determined to belong to the two species Cacopsylla melanoneura and Cacopsylla affinis; -, water; M, Gene Ruler ${ }^{\mathrm{TM}} 100$ bp DNA Ladder (Fermentas).

Following confirmation of amplicon sequence and length by direct sequencing, this was deemed an artefact of migration associated with the peculiar base composition and secondary structures of the amplified fragments. The test, performed on 52 individuals of both species that had been morphologically determined to the species level, including 12 for which the complete sequence of the CR had been previously obtained, gave $100 \%$ success in assigning the species correctly. Based on amplicon length, individuals of $C$. melanoneura could be clearly identified as having or not having the $56 \mathrm{bp}$ indel, and this was consistent with previously obtained sequence data. For simplicity, the genetic variants with and without the indel were named WI and WOI, respectively.

\section{Population of C. melanoneura-C. affinis complex collected on hawthorn plants}

Screening results from C. melanoneura-C. affinis mixed samples of overwintered individuals, collected on Cr. monogyna plants from the Aosta Valley, are shown in table 1. C. melanoneura is clearly the predominant species and accounts for $86.5 \%$ (80.4-91.2, 95\% confidence interval (CI)) of captured psyllids, but the presence of C. affinis $(13.5 \%$; 8.8-19.6 CI) was nevertheless considerable. Fluctuations across the two years of collection were limited.

Individuals of $C$. melanoneura belonging to both genetic variants were found, with WOI being the predominant genotype and accounting for $81.6 \%$ (74.4-87.5 CI) of captured C. melanoneura. Again, this figure was stable across years. 
Table 1. Species and genetic variant frequency in overwintered Cacopsylla melanoneura-Cacopsylla affinis mixed samples on hawthorn from the Aosta Valley.

\begin{tabular}{lccccc}
\hline Year & $\begin{array}{c}\text { Total } \\
\text { sampled }\end{array}$ & \multicolumn{3}{c}{ C. melanoneura $\%$} & \multirow{2}{*}{ C. affinis \% } \\
\cline { 3 - 5 } & & Total C. mel. $\%$ & WI \%* & WOI \%* & \\
\hline 2006 & 110 & 89.1 & 18.4 & 81.6 & 10.9 \\
& & $(81.7-94.2)$ & $(11.3-27.5)$ & $(72.5-88.7)$ & $(5.8-18.3)$ \\
2008 & 60 & 81.7 & 18.4 & 81.6 & 18.3 \\
& & $(69.6-90.5)$ & $(8.8-32.0)$ & $(68.0-91.2)$ & $(9.5-30.4)$ \\
Total per species and & 170 & 86.5 & 18.4 & 81.6 & 13.5 \\
genetic group & & $(80.4-91.2)$ & $(12.5-25.6)$ & $(74.4-87.5)$ & $(8.8-19.6)$ \\
\hline
\end{tabular}

* WI and WOI variants' frequency are calculated on the total number of $C$. melanoneura specimens.

$95 \% \mathrm{CI}$ are shown in brackets.

Table 2. Frequency of the two Cacopsylla melanoneura genetic variants in samples collected on apple plants from different locations.

\begin{tabular}{lccc}
\hline Site & \multicolumn{3}{c}{ C. melanoneura } \\
\cline { 2 - 4 } & $\begin{array}{c}\text { Total } \\
\text { sampled }\end{array}$ & WI \% & WOI \% \\
\hline Aosta Valley & 21 & 9.5 & 90.5 \\
& & $(1.2-30.4)$ & $(69.6-98.8)$ \\
Valsugana & 20 & 5.0 & 95.0 \\
& & $(0.1-24.9)$ & $(75.1-99.9)$ \\
Val di Non & 18 & 5.6 & 94.6 \\
& & $(0.1-27.3)$ & $(72.7-99.9)$ \\
Alto Adige & 16 & 31.3 & 68.7 \\
& & $(11.0-58.7)$ & $(41.3-89.0)$ \\
Canavese & 24 & 8.3 & 91.7 \\
& & $(1.0-27.0)$ & $(73.0-99.0)$ \\
Valtellina & 22 & 18.2 & 81.8 \\
& & $(5.2-40.3)$ & $(59.7-94.8)$ \\
\hline
\end{tabular}

95\% CI are shown in brackets.

\section{Population of C. melanoneura collected on apple plants}

The screening of specimens collected from apple plants in six locations in northern Italy (table 2), all confirmed as to belonging to $C$. melanoneura, led to the identification of both WI and WOI genetic groups in all locations, with WOI group predominant overall (87.6\%; 80.4-93.0 CI). Some geographic differences in genotype frequency were observed. WI haplotypes were found at low frequency in some locations (5.0\% (0.1-24.9 CI) in Valsugana and 5.6\% (0.1-27.3 $\mathrm{CI})$ in Val di Non) and at higher frequency in others $(18.2 \%$ (5.2-40.3 CI) in Valtellina and 31.3\% (11.0-58.7 CI) in Alto Adige). Nevertheless, a $\chi^{2}$ test could not reject the null hypothesis of uniformity of genotypes across locations $\left(\chi^{2}=8.22\right.$; $\mathrm{df}=5 ; P=0.14$ ), possibly as a consequence of the limited number of samples.

\section{Phytoplasma detection}

In the mixed sample of $C$. melanoneura (variants WI and WOI) and C. affinis sampled on hawthorn, the overall frequency of phytoplasma-positive psyllids was very low (four out of 161 samples, corresponding to $2.5 \%$, overall). In the $C$. melanoneura group WOI, one individual out of
116 (6.2\%) was found positive for the 16SrX-A profile, corresponding to ' $\mathrm{C} a$. Phytoplama mali', while two out of $116(1.7 \%)$ individuals of $C$. melanoneura group WOI and one out of $21(4.8 \%)$ of $C$. affinis were found positive for profile 16SrX-C, corresponding to 'Ca. Phytoplama pyri'. 'Ca. Phytoplasma prunorum' was never detected in our samples.

The same analysis was performed on the samples of C. melanoneura variants WI and WOI shown in table 2; the individuals collected on apple in Canavese displayed a slightly higher frequency of phytoplasma infections (six out of 24, 25\%), with five out of $24(20.8 \%)$ instances of 'Ca. Phytoplama mali' and one out of $24(4.2 \%)$ of ' $\mathrm{Ca}$. Phytoplasma pyri' all in individuals belonging to the genetic variant WOI. In the samples collected in Valtellina, ' $\mathrm{Ca}$. Phytoplasma mali' was found in two out of $22(9.1 \%)$ specimens belonging to the genetic variant WOI. In no other sample was AP-group phytoplasma detected.

The molecular test, performed on 63 C. melanoneura specimens previously proven to be infected with ' $\mathrm{Ca}$. Phytoplasma mali', identified that most infected individuals belong to the WOI genotype, with only six (10\%) identified WI genotypes.

\section{Discussion}

The present work describes a new DNA-based tool for the species-level discrimination of $C$. melanoneura and C. affinis by means of PCR without requiring the support of a specialist for morphological determination. Furthermore, the test is applicable to all life stages and is the only possible way to determine species identity of females, which cannot be distinguished based on morphology. This new test will allow the study of the life cycle of $C$. affinis, as well as its copresence with $C$. melanoneura on host plants and its possible role as a vector of AP phytoplasmas, in more detail.

In a previous study (Tedeschi et al., 2009), based solely on morphological species discrimination of males, it was shown that C. affinis accounted for $21.6 \%$ (19.6-23.7 CI) of the overwintered population and $6.9 \%(3.5-12.0 \mathrm{CI})$ of newlyemerged adults, suggesting a small time difference in the biological cycle of the two species (confidence intervals calculated based on the original data from Tedeschi et al. (2009)). The present analysis, where both sexes were taken into consideration, produced an intermediate value (13.5; 8.8-19.6 CI), suggesting a role of females in the different proportion of the two species. Further studies are now 
necessary to better define the proportion of the two species on Cr. monogyna year round. These studies will allow to determine whether life cycles of the two species are synchronous, or if there is a time-lag in their biology.

The conjunct application of the two tests for species discrimination and phytoplasma detection showed that C. affinis can acquire AP-group phytoplasmas, and in particular 'Ca. Phytoplasma pyri', while Tedeschi et al. (2009) already reported (on a sample of males only) the ability of this species to acquire ' $\mathrm{Ca}$. Phytoplasma prunorum'. This work confirms the ability of $C$. affinis to acquire fruit tree phytoplasma, but now transmission trials are required to verify this species' ability to function as a vector. The ability to identify C. affinis using a PCR based-test will make those trials more straightforward.

Furthermore, initial sequencing revealed the presence of two distinct genetic profiles in C. melanoneura, here named WI and WOI, that differ by the presence/absence of a $56 \mathrm{bp}$ indel. Although the origin and geographic distribution of such variants is yet to be investigated in detail, this opens the possibility of an initial assessment of population genetic variation within C. melanoneura and of testing for the existence of differences in host preferences, frequency of phytoplasma, and propensity to transmit it to host plants.

Interestingly, transmission trials carried out to assess the role of $\mathrm{C}$. melanoneura as vector for ' $\mathrm{C}$. Phytoplasma mali' gave inconsistent results when conducted with specimens from different geographic origins. Although $C$. melanoneura represents the main vector of 'Ca. Phytoplasma mali' in northwestern Italy, low transmission efficiency was reported in northeastern Italy (Mattedi et al., 2008), and no transmission was observed in Germany (Jarausch et al., 2007; Mayer et al., 2009). These discrepancies might be due to the presence of different phytoplasma strains in different geographic regions that differ in their ability to be transmitted and infect host plants, but the possibility of different C. melanoneura variants with different phytoplasma transmission efficiencies cannot be excluded a priori.

The identification of the WI and WOI genotypes suggested a preliminary investigation of C. melanoneura specimens coming from different Italian regions, in which different levels of transmission efficiency had been described and, in turn, led to the observation that different proportions of specimens belonging to the two groups were collected in these different regions. Similar higher proportions of WI genotypes were recorded in the Aosta Valley and in Canavese, where good transmission efficiency has been recorded (Tedeschi \& Alma, 2004). Conversely, the percentages of $C$. melanoneura genotypes WI recorded in Valsugana and Val di Non, where low transmission efficiency was recorded (Mattedi et al., 2008), appears to be lower. These preliminary observations open the possibility of a correlation between $C$. melanoneura genetic variants and their tendency to transmit phytoplasma. Nevertheless, although the pattern observed in our data is intriguing, the statistical analysis fails to reject the null hypothesis of uniformity across locations. A thorough testing on a higher number of samples, as well as cage studies, will be needed to assess this possibility in some detail before any conclusion can be drawn. Furthermore, the molecular test carried out with MEL_fw/MEL_rev on 63 C. melanoneura specimens previously proven to be infected with 'Ca. Phytoplasma mali' demonstrates that both WI and WOI variants can carry the phytoplasma.
Further investigations of the relative roles of the two species in phytoplasma transmission and on the correspondence between $C$. melanoneura genotype and host plant choice would be highly valuable in terms of the epidemiology of apple proliferation disease.

In a related system, Sauvion et al. (2007) described two highly differentiated populations of Cacopsylla pruni (Scopoli), the psyllid vector of 'Ca. Phytoplasma prunorum' that occur sympatrically in most sites studied, again leading to a question about their relative ' $\mathrm{Ca}$. Phytoplasma prunorum' vector efficiency. Among phytoplasma insect vectors, this is paralleled by the cixiid planthopper Hyalesthes obsoletus Signoret, where recent analyses carried out by Johannesen et al. (2008) suggested the possible existence of host-races of both the vector and the phytoplasma.

Finally, although our DNA-based test fully succeeded in the validation procedure applied here, it is important to note that the background information on which the test is designed (sequences of the CR of the two species) is limited to northwestern Italy, and validation of the test was based on additional samples from the same area. Expanding the use of this methodology to additional areas and populations, or the use of the indel's presence/absence as a population genetic marker, will require additional sequencing and testing of local material; mutations in primer binding sites, as well as duplications, large indels and heteroplasmy, are rather frequent in the insect $\mathrm{CR}$ and might complicate or impair the tests.

\section{Acknowledgements}

We are grateful to Dr Josef Österreicher from the Südtiroler Beratungsring für Obst-und Weinbau, Lana (BZ), Dr Valeria Malagnini, Dr Federica Trona and Dr Mario Baldessari from IASMA-FEM, San Michele all'Adige (TN) and Dr Paola Casati from Dipartimento di Produzione vegetale (Sezione di Patologia Vegetale) - Università degli Studi di Milano for psyllid collections. We thank Dr Pavel Lauterer from the Moravian Museum, Brno (Czech Republic) for his help in the identification of psyllid species. We also thank Dr Maggie Sherriffs from the University of California, Davis for the English revision of the manuscript.

\section{References}

Čermák, V. \& Lauterer, P. (2008) Overwintering of psyllids in south Moravia (Czech Republic) with respect to the vectors of the apple proliferation cluster phytoplasmas. Bulletin of Insectology 61, 147-148.

Frisinghelli, C., Delaiti, L., Grando, M.S., Forti, D. \& Vindimian, M.E. (2000) Cacopsylla costalis (Flor, 1861), as a vector of apple proliferation in Trentino. Journal of Phytopathology 148, 425-431.

Jarausch, B., Schwind, N., Jarausch, W., Krczal, G., Seemüller, E. \& Dickler, E. (2003) First report of Cacopsylla picta as a vector for apple proliferation phytoplasma in Germany. Plant Disease 87, 101.

Jarausch, B., Fuchs, A., Schwind, N., Krczal, G. \& Jarausch, W. (2007) Cacopsylla picta as most important vector for 'Candidatus Phytoplasma mali' in Germany and neighbouring regions. Bulletin of Insectology 60, 189-190.

Johannesen, J., Lux, B., Michel, K., Seitz, A. \& Maixner, M. (2008) Invasion biology and host specificity of the 
grapevine yellows disease vector Hyalesthes obsoletus in Europe. Entomologia Experimentalis et Applicata 126, 217-227.

Lal, K.B. (1934) The biology of Scottish Psyllidae. Transactions of the Royal Entomological Society of London 82, 363-385.

Lauterer, P. (1999) Results of the investigations on Hemiptera in Moravia, made by the Moravian museum (Psylloidea 2). Acta Musei Moraviae (Scientiae biologicae), Brno 84, 71-151.

Lorenz, K.H., Schneider, B., Ahrens, U. \& Seemüller, E. (1995) Detection of the Apple Proliferation and Pear Decline Phytoplasmas by PCR amplification of ribosomal and non ribosomal DNA. Phytopathology 85, 771-776.

Marzachì, C., Veratti, F. \& Bosco, D. (1998) Direct PCR detection of phytoplasmas in experimentally infected insects. Annals of Applied Biology 133, 45-54.

Mattedi, L., Forno, F., Cainelli, C., Grando, M.S. \& Jarausch, W. (2008) Research on Candidatus Phytoplasma mali transmission by insect vectors in Trentino. Acta Horticulturae 781, 369-374.

Mayer, C.J. \& Gross, J. (2007) Different host plant odours influence migration behaviour of Cacopsylla melanoneura (Förster), an insect vector of the apple proliferation phytoplasma. IOBC/WPRS Bulletin 30(4), 177-184.

Mayer, C.J., Jarausch, B., Jarausch, W., Jeklmann, W., Vilcinskas, A. \& Gross, J. (2009) Cacopsylla melanoneura has no relevance as vector of apple proliferation in Germany. Phytopathology 99, 729-738.

Ossiannilsson, F. (1992) The Psylloidea (Homoptera) of Fennoscandia and Denmark. Fauna Entomologica Scandinava 26, 1-347.
Sauvion, N., Lachenaud, O., Genson, G., Rasplus, J.-Y. \& Labonne G. (2007) Are there several biotypes of Cacopsylla pruni? Bulletin of Insectology 60, 185-186.

Schneider, E., Seemüller, E., Smart, C.D. \& Kirkpatrick, B.C. (1995) Phylogenetic classification of plant pathogenic mycoplasma-like organism or phytoplasmas. pp. 369-380 in Razin, S., Tully, J.G. (Eds). Molecular and Diagnostic Procedures in Mycoplasmology. vol I. San Diego, CA, USA, Academic Press.

Tedeschi, R. \& Alma, A. (2004) Transmission of apple proliferation phytoplasma by Cacopsylla melanoneura (Homoptera: Psyllidae). Journal of Economical Entomology 97, 8-13.

Tedeschi, R. \& Alma, A. (2006) Fieberiella florii (Homoptera: Auchenorrhyncha) as a vector of 'Candidatus Phytoplasma mali'. Plant Disease 90, 284-290.

Tedeschi, R., Bosco, D. \& Alma, A. (2002) Population dynamics of Cacopsylla melanoneura (Homoptera: Psyllidae), a vector of apple proliferation in northwestern Italy. Journal of Economic Entomology 95, 544-551.

Tedeschi, R., Visentin, C., Alma, A. \& Bosco, D. (2003) Epidemiology of apple proliferation (AP) in northwestern Italy: evaluation of the frequency of AP-positive psyllids in naturally infected populations of Cacopsylla melanoneura (Homoptera Psyllidae). Annals of Applied Biology 142, 285290.

Tedeschi, R., Lauterer, P., Brusetti, L., Tota, F. \& Alma, A. (2009) Composition, abundance and phytoplasma infection in the hawthorn psyllid fauna of northwestern Italy. European Journal of Plant Pathology 123, 301-310. 